\title{
Design and Implementation of Air Quality Monitoring System based on Lab-View and Related Techniques
}

\author{
LI Danian ${ }^{1,}$, ZHAI Chongzhi' ${ }^{2}$ YU Jiayan ${ }^{3}$, BAO Lei ${ }^{4}$ \\ 1,2,3,4 Key laborary for urban atmospheric integrated observation \& pollution prevention and control of \\ Chongqing, Chongqing Municipal Environmental Monitoring Center ,Chongqing

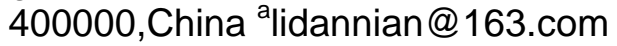

Keywords: Air Quality Monitoring System; Lab-View; Risk Analysis; Embed System Monitoring.

\begin{abstract}
With STC89C51 as the core controller, the distributed wireless monitoring system for air quality has been designed and implemented in this paper. With the improvement of human material civilization, increasing economic benefits, the nature of the human damage is aggravating. Environmental pollution is an urgent and problems to be solved, and the sustainable development of mankind. According to relevant data show that China's air pollution is very serious. Satisfy the standard of national air quality level two city accounts for only a third, more than triple the standard cities also accounted for almost a third. Through the usage of Matlab and Lab-View, we implement the current system with the analysis of parameter estimation and test. The simulation result shows that our system is more robust and accurate compared with other state-of-the-art systems.
\end{abstract}

\section{Introduction}

Indoor air quality parameters include impacts on human health of physical, chemical, biological and radioactive and inhalable related particle parameters. The pros and cons of indoor air quality, more and more influences on human health. Traditional air quality monitoring instruments mainly has two kinds: one is to use a handheld manual monitoring instrument, the instrument there exist large workload, low efficiency, disadvantage; the second is to use more cable transmission instrument for monitoring, the method is poor system scalability, wiring and trival, price higher shortcoming. In recent years, some domestic enterprises and personnel made wireless air quality monitoring system of research and development, but the price of these products are expensive, popularity is difficult, and without considering the space limitations in some industries and places and operator level of the actual situation of applicability is poor, has not been widely used [1-3]. With the improvement of human material civilization, increasing economic benefits, the nature of the human damage is aggravating. Environmental pollution is an urgent and problems to be solved, and the sustainable development of mankind. According to relevant data show that China's air pollution is very serious. Satisfy the standard of national air quality level two city accounts for only a third, more than triple the standard cities also accounted for almost a third. According to world bank statistics, our country of the city's total suspended particulate matter and sulfur oxides are more than the world health organization (who) standards [4-9]. The current method of air quality monitoring is mainly operating personnel with the monitoring equipment to the site sampling, then samples to laboratory or environmental monitoring center for analysis. For some harsh environment testing station, dangerous, it is not suitable for long-term testing, and could not reach the requirement of real-time monitor, so the real-time automatic monitoring system of air environmental quality will be the future trend of the development of environmental monitoring. Large building is turnover more public places, especially the staff to work on this for a long time, poor liquidity of air inside the building, directly endanger people's health. Due to the improvement of living standards and the whole society's awareness of environmental protection, to the life, work environment air quality requirements also more and more high. Therefore, in the large building it is necessary to install air quality monitoring system, through the office environment air quality monitoring, take appropriate measures, can reduce the threat to health, to improve the efficiency of office will play a very important role. In view of the large building environment within the specific characteristics of wireless sensor network technology is 
applied in this article, based on the feature of the fast, flexible control and PC powerful monitoring and management functions, design a kind of air quality monitoring system, implementation of data acquisition, transmission, monitoring and management.

In this article, we will in the wireless communication technology is applied to air quality monitoring, air quality from various sensors collect real-time measurement point of information. Air quality information after processing, through the wireless data acquisition system is transmitted to the monitoring terminal. The operator can choose test points in the handset, after processing the received data in handset LCD screen display, so as to realize the goal of integration, low cost and easy operation. Through the implementation together with the lab-view platform, we verify the robustness and effectiveness of the proposed approach.

\section{Design and Implementation of the System}

The General Structure of the Sensor System. Assessing the sensor's potential is done by factoring in expected system performance, and also via experimental verification. System is composed with a host with multiple from host mainly include microprocessors, wireless receiving module, liquid crystal display module, keys module and alarm module. From the machine, through the smoke sensor simulation of smoke concentration can be converted to electrical signals, then through high speed A/D converter converts analog signals quickly to microcontroller available digital signal. Micro controller through simulation SPI bus to the wireless data transmission module, wireless module and the data sent. On a host, the wireless module in the receiving state is waiting to take the machine to send data. When detected from machine data and carrier frequency and address after the match, the host wireless module began accepting data sent from the machine. After receiving the data, wireless modules nRF905 automatically remove lead code and CRC check code, the effective data is stored in the receiving register. Microprocessor simulation SPI bus from reading the data register of nRF905 read from the machine to send data, complete the wireless data communication. Host receives the data after data processing into the corresponding gas sensitivity which calls a serial LCD procedure and shows that gas sensitivity parameters on the LCD. Because the system is a distributed air quality monitoring system, the host to monitoring air quality, several measurement points as a result, the host must establish communication with multiple from the machine. Different from the machine is equipped with its own address, host and from the machine set up by means of time-sharing query address communication, receive the data from the machine. Key modules can be installed air sensitivity of the monitoring alarm threshold. When a monitoring air pollution levels, the system will automatically start the buzzer alarm circuit, and sends an alarm. The basic Radio Frequency (RF) components of the laboratory prototype are available off-the-shelf. The block diagram of the sensor discussed below is shown in Fig. 1. The key element of the sensor is a Voltage-Controlled Oscillator (VCO) modulated by a triangular wave signal.

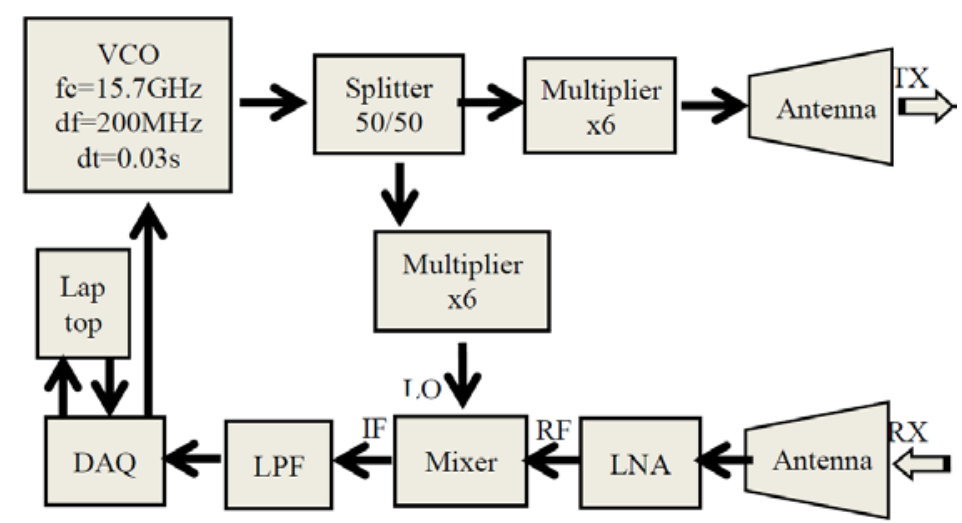

Figure 1.The Structure of the Sensor System

The Hardware Design and Analysis. Mainly by the wireless gateway nodes send/receive module, embedded processors, memory and other parts, main function is to deal with and send the collection 
of data. Gateway nodes connected to the sensor network and monitoring center computer, respectively, are still using chip nRF905, can be unified transport protocol, ensure the reliability of the transmission, but also due to data processing, the gateway node is not additional sensors, in order to improve the ability of data processing. On the one hand it is responsible for receiving sensor network gateway nodes. From the data and process for correction, fusion, etc. and sent to the monitoring center computer, on the other hand to send command receiving center monitor computer for monitoring center, by the corresponding processing instructions, release monitoring task. Sensor nodes working process mainly include self-checking system, data acquisition module, data receiving and sending part, power management, etc. [10-12]. Start the program after the system is powered on, the port is configured, using interrupt way to execute the corresponding module is the main purpose of the gateway node to receive data from the air sensor nodes, the corresponding processing and fusion, and sent to the monitoring center computer; Receive instruction monitoring center, to determine the node working state monitoring personnel through monitoring software, can be real-time observed air quality data, using monitoring software can also form database, report forms, on the basis of monitoring data, use a chart, curve display or printing software is mainly composed of the menu interface, database operation interface, communication interface and so on three modules. Specific design is in $\mathrm{C}++$ Builder integrated development environment, the window of the newly built based on the visual elements (VCL) application, project called SCOMMN, all steps to keep the default state, and add controls in the main form. The structure is shown in the figure 2.

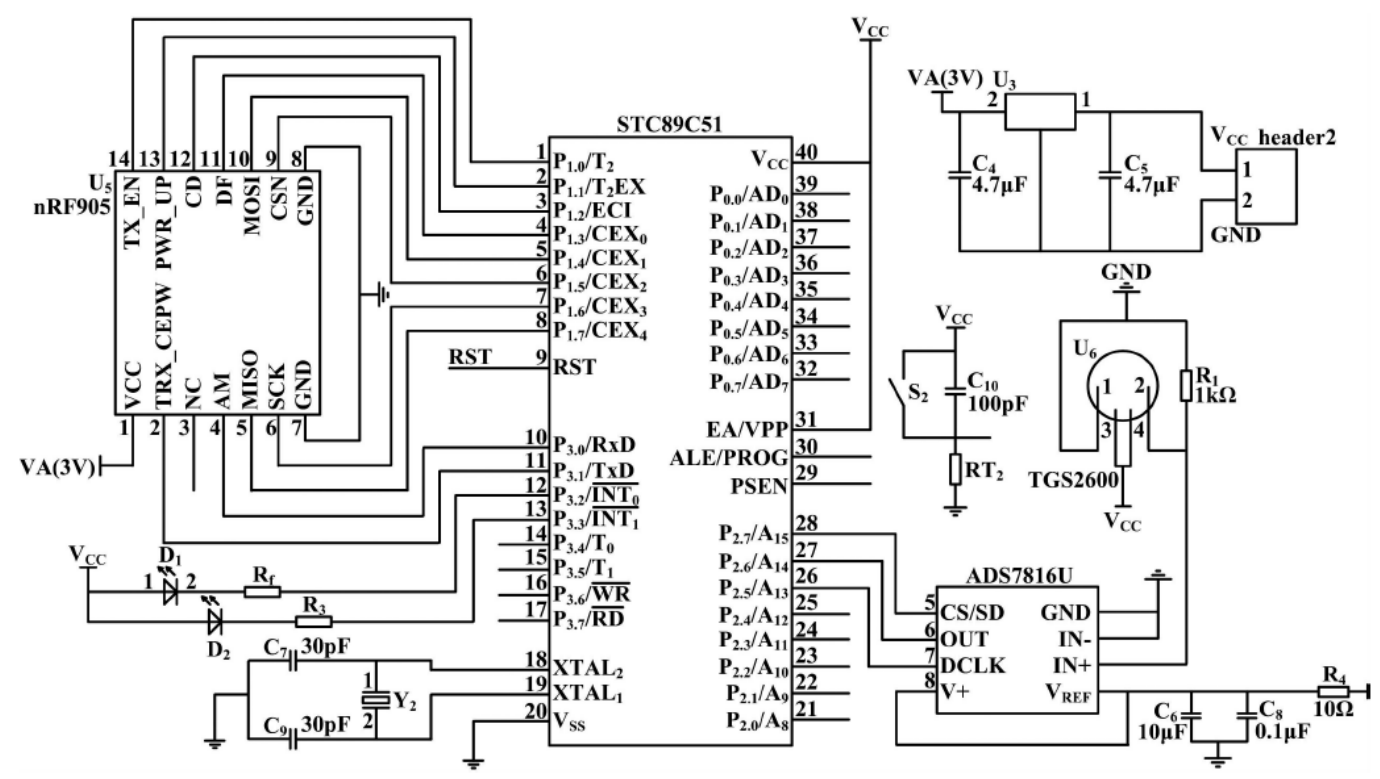

Figure 2.The Hardware Design of the System

The Mathematical Analysis and Data Acquisition. We conduct mathematical and numerical analysis for the parameters of the system. Noise performance and minimum detectable signal is considered to be a most important standard. The received power can be estimated using the radar equation and the expression could be concluded to be the formula 1.

$$
F_{T O T, \text { Max }}=F_{a m p, \text { Max }}+\frac{\left(C L_{\text {Mix }}-1\right)}{G_{a m p, \min }}+\frac{\left(F_{L P F, M a x}-1\right) C L_{\text {Mix }}}{G_{a m p, \min }}
$$

Using the values for the minimum received power and the noise floor, the signal-to-noise ratio (SNR) at $60 \mathrm{~m}$ is obtained as $49.17 \mathrm{~dB}$. The distance of $60 \mathrm{~m}$ was selected as this detection distance allows not only for a warning but also for an automatic slowing down, and even for a complete halting of the vehicle. From this calculation it emerges that detection at even larger distances is feasible under these terms. Mutual coupling between antennas can be a source of parasitic leakage causes degradation of the overall system noise performance, saturated front-end low-noise amplifier. The distance between the coupling effect depends on the polarization and antenna shown in the figure 3 . The interface for the system is shown in the figure 4 . 

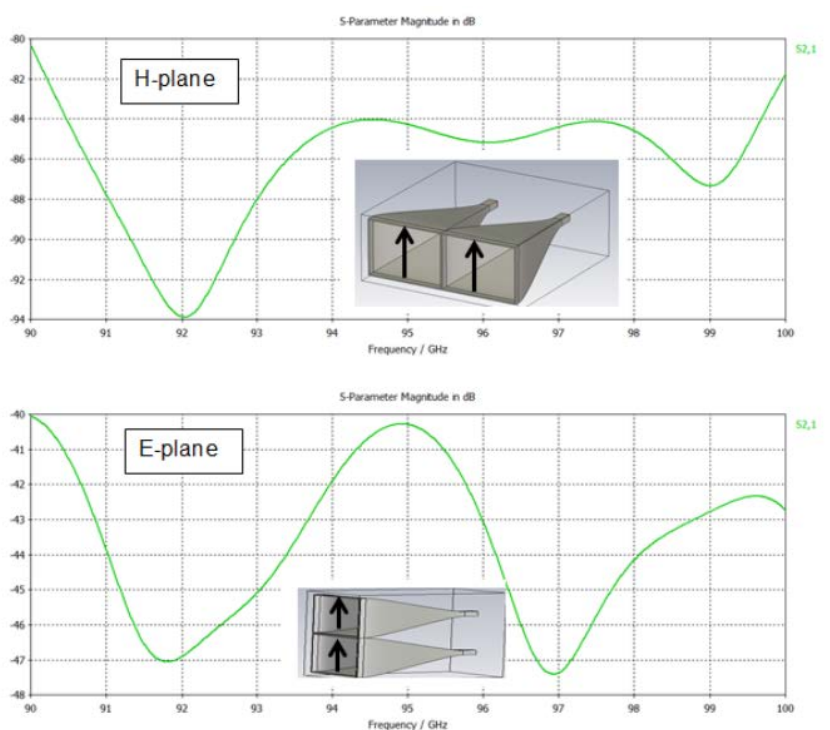

Figure 3.The Distance between the Coupling Effect

\begin{tabular}{|c|c|}
\hline $\mathrm{AT} 89 \mathrm{C} 51$ & nRF905 \\
\hline P1.0 & TX_ENP \\
\hline P1.1 & WR_UP \\
\hline P3.4 & TRX_CE \\
\hline P1.3 & CSN \\
\hline P1.7 & SCK \\
\hline P1.5 & MOSI \\
\hline P1.6 & MISO \\
\hline P1.2 & $\mathrm{CD}$ \\
\hline P1.4 & AM \\
\hline P3.2 & $\mathrm{DR}$ \\
\hline
\end{tabular}

Figure 4.The Interface for the Proposed System

The General Overview for the Test. System software in several important sub-routines for analog-to-digital conversion program, nRF905 wireless module and LCD screen display program. From the machine software is mainly divided into data acquisition module and data sending module. Among them, the data acquisition module is mainly to complete the data acquisition and data processing, data sending module implements the wireless transmission of data gathering. Based on Zig Bee large building air quality monitoring network, design the system hardware and software, can real-time display the monitoring data, to refresh regularly, historical data displayed in a list form. In view of the low-power wireless design, data transmission signals are relatively weak, poor anti-interference ability, easy to data leakage phenomenon, by adopting the method of software improvement, increase the frequency of data collection, extend the data transfer cycle, reduce the amount of data packet loss rate. Operation through the experiment testing and monitoring parameters, the system of CO, formaldehyde, benzene building environment air quality parameters, such as SO2 and the real-time monitoring function can achieve reliable, convenient extension of the system, through the external different sensors, the system can monitor various environmental parameters.

\section{Experiment and Simulation Result}

Experiments were conducted with the abovementioned radar in various environments and targets. Firstly, range calibration measurements were taken with a corner reflector to verify the radar's range readings. Then, both adult and child measurements were taken in a 'road-like' environment, and in a grassy soccer field. Measurements were taken in real time using the LabView, and also, post processing was done using Matlab. Several rounds of experiments were conducted and the 
conclusions from the MATALAB analysis were implemented into the LabView code to enhance the real time algorithm. The experimental user interface is shown in the figure 5.

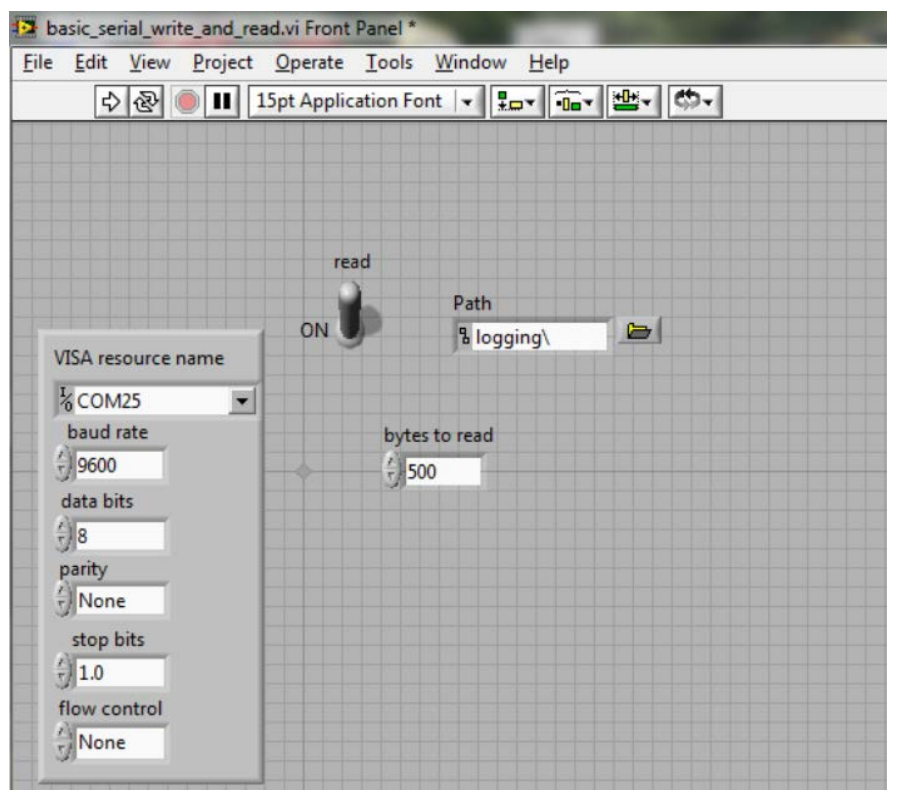

Figure 5.The User Interface for the System

Tests, the use of board design A/D conversion module, liquid crystal display module, the singlechip processor system and wireless transceiver module circuit, module debugging independently, the final alignment. Test steps are as follows: using altium designer map system principle, the corresponding components of pin and drawing system packaging figure; Draw PCB carving, carved PCB for circuit testing, to ensure that the correct welding components on the PCB board after; Loading system code, whether detection system run normally, if a runtime error, to continue to check the hardware circuit, until the system to work normally. The experiment result is shown in the figure 6.

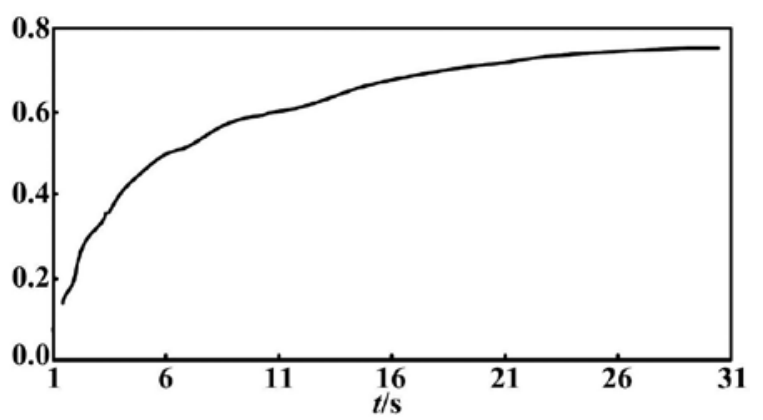

Figure 6.The Final Result for the System Test

\section{Conclusion and Summary}

Indoor air quality parameters include impacts on human health of physical, chemical, biological and radioactive and inhalable related particle parameters. In recent years, some domestic enterprises and personnel made wireless air quality monitoring system of research and development, but the price of these products are expensive, popularity is difficult, and without considering the space limitations in some industries and places and operator level of the actual situation of applicability is poor, has not been widely used. With the improvement of human material civilization, increasing economic benefits, the nature of the human damage is aggravating. We use the star topology network, through the monitoring area deployment of network nodes, the monitoring data to the embedded monitoring system which realize the unified data management and Zig Bee network routing monitoring function. Environmental pollution is an urgent and problems to be solved, and the sustainable development of 
mankind. According to relevant data show that China's air pollution is very serious. Therefore, we conduct research on air quality monitoring system based on Lab-View and related techniques in this paper. Through the implementation together with the lab-view platform, we verify the robustness and effectiveness of the proposed approach.

\section{Acknowledgements}

The major national scientific instrument development and special project (2012YQ060147) $<<$ fine particles in ambient air (PM2.5) development and application of monitoring equipment $>>$.

\section{References}

[1] Yang, Chao-Tung, et al. "Construction and application of an intelligent air quality monitoring system for healthcare environment." Journal of medical systems 38.2 (2014): 1-10.

[2] Wong, Man Sing, Tsan Pong Yip, and Esmond Mok. "Development of a Personal Integrated Environmental Monitoring System." Sensors 14.11 (2014): 22065-22081.

[3] Shaposhnikov, Dmitry, Boris Revich, Tom Bellander, Getahun Bero Bedada, Matteo Bottai, Tatyana Kharkova, Ekaterina Kvasha et al. "Mortality related to air pollution with the Moscow heat wave and wildfire of 2010." Epidemiology (Cambridge, Mass.) 25, no. 3 (2014): 359.

[4] Moreno, T., N. Pérez, C. Reche, V. Martins, E. de Miguel, M. Capdevila, S. Centelles et al. "Subway platform air quality: Assessing the influences of tunnel ventilation, train piston effect and station design." Atmospheric Environment 92 (2014): 461-468.

[5] Kim, Seulki, and Seung-Hoon Han. "Establishment of the Monitoring System for Human Comfort Towards Traditional Residential Environment." Journal of Korean Institute of Information Technology 13, no. 1 (2015): 149-155.

[6] Hamaoui-Laguel, Lynda, Frédérik Meleux, Matthias Beekmann, Bertrand Bessagnet, Sophie Génermont, Pierre Cellier, and Laurent Létinois. "Improving ammonia emissions in air quality modelling for France." Atmospheric Environment 92 (2014): 584-595.

[7] Rajasegarar, Sutharshan, et al. "High-resolution monitoring of atmospheric pollutants using a system of low-cost sensors." Geoscience and Remote Sensing, IEEE Transactions on 52.7 (2014): 3823-3832.

[8] Hache, E., J-L. Attié, C. Tourneur, P. Ricaud, L. Coret, W. A. Lahoz, L. El Amraoui et al. "The added value of a visible channel to a geostationary thermal infrared instrument to monitor ozone for air quality." Atmospheric Measurement Techniques 7, no. 7 (2014): 2185-2201.

[9] Lederer, Johannes, and Sara Van De Geer. "New concentration inequalities for suprema of empirical processes." Bernoulli 20, no. 4 (2014): 2020-2038.

[10]Zoogman, Peter, et al. "Monitoring high-ozone events in the US Intermountain West using TEMPO geostationary satellite observations." Atmospheric Chemistry and Physics 14.12 (2014): 6261-6271.

[11]Kumar, Anuj, and Gerhard P. Hancke. "Energy efficient environment monitoring system based on the IEEE 802.15. 4 standard for low cost requirements." (2014): 1-1.

[12] Viana, M., J. Pey, X. Querol, A. Alastuey, F. De Leeuw, and Anke Lükewille. "Natural sources of atmospheric aerosols influencing air quality across Europe." Science of the Total Environment 472 (2014): 825-833.

[13]Weidong zhang,Ming chen "PAHs content and its distribution in the downtown area of Chongqing city in different diameter airborne particles. " Environmental impact assessment(2013)1(1)::55-59 